\title{
Evaluación de competencias. Retos y perspectivas desde el nivel básico al universitario
}

\author{
Evaluation of competences. Challenges and perspectives from \\ basic to university level
}

Manuel F. Villero ${ }^{\square}$ (D) Regina Agramonte ${ }^{2}$ (D) Eduardo Menéndez-Álvarez ${ }^{3}$

\footnotetext{
${ }^{1}$ Universidad Metropolitana de Educación, Ciencia y Tecnología. Ciudad de Panamá. Panamá

${ }^{2}$ Universidad Científica del Sur. Lima, Perú

${ }^{3}$ Universidad Le Cordon Bleu. Lima, Perú
}

Recibido: 05/08/2021

Revisado: 20/09/2021

Aceptado: 01/12/2022

Publicado: 15/01/2022

\section{RESUMEN}

El propósito central de la investigación fue analizar la evaluación de competencias, retos y perspectivas desde el nivel básico al universitario. Se fundamentó teóricamente en los postulados de expertos, quienes desde su perspectiva permiten contrastar sus diferentes concepciones sobre la temática en cuestión. Metodológicamente se trata de un estudio asociado al paradigma cualitativo de corte exploratorio, bajo la modalidad documental con diseño bibliográfico. Entre los hallazgos más relevantes de la investigación se develó que el enfoque curricular basado en competencias permite abrir rutas al camino de la profesionalización, desde una perspectiva epistemológica y paradigmática que facilita la generación de políticas y cambios en el diseño curricular y su puesta en práctica, tomando en cuenta aspectos relevantes en el proceso como las inversiones humanas, materiales y financieras de las instituciones, siendo la pieza central del enfoque curricular los perfiles de egreso, buscando desarrollar habilidades específicas y genéricas en los estudiantes, indistintamente del nivel educativo que cursen. Se propone un enfoque de evaluación constructivista, contextual basado en competencias, lo cual implica centrar la praxis educativa en un modelo evaluativo orientado a los procesos y pasos de los pilares del saber fundamentado en el desarrollo integral de los estudiantes. Palabras clave: Evaluación por competencias, educación básica, educación universitaria, currículo por competencia, praxis educativa.

\section{ABSTRACT}

The main purpose of the research was to analyze the evaluation of competencies, challenges and perspectives from the basic to the university level. It was theoretically based on the postulates of experts, who from their perspective allow contrasting their different conceptions on the subject in question. Methodologically, it is a study associated with the qualitative exploratory paradigm, under the documentary modality with bibliographic design. Among the most relevant findings 
of the research, it was revealed that the competency-based curricular approach allows opening routes to the path of professionalization, from an epistemological and paradigmatic perspective that facilitates the generation of policies and changes in the curriculum design and its implementation, taking into account relevant aspects in the process such as human, material and financial investments of the institutions, being the centerpiece of the curricular approach the graduation profiles, seeking to develop specific and generic skills in students, regardless of the educational level they are studying. A constructivist, contextual, competency-based evaluation approach is proposed, which implies focusing educational praxis on an evaluative model oriented to the processes and steps of the pillars of knowledge based on the integral development of students.

Keywords: Competency assessment, basic education, university education, competency curriculum, educational praxis.

\section{INTRODUCCIÓN}

El desarrollo en todos los ámbitos de la sociedad, sumado al avance tecnológico y a la pandemia del Covid-19, han traído consigo la necesidad de introducir cambios en el ámbito educativo con propuestas de nuevos paradigmas; y en consecuencia, el uso de diferentes medios y técnicas dentro y fuera del aula, plantean nuevas exigencias, demandando concebir de manera diferente y creativa la evaluación de la praxis del sistema educativo y su repercusión en el desarrollo de habilidades de los estudiantes. En este contexto la ciencia y la tecnología, para la educación, además, la gestión e impacto en la sociedad, son vistos como elementos esenciales en el desarrollo socioeconómico, científico, tecnológico y político de las naciones, aunado al papel protagónico de la educación, encaminando el progreso de éstas.

Rojas (2016, p.24) plantea que "la educación es el activo más importante de una sociedad", permitiendo diseñar modelos de política económica y social que sirvan de mecanismos para el desarrollo de competencias, que propicie afrontar la globalización y obtener mayores beneficios posibles cónsonos con la realidad.
A juicio de Leboyer (2002, p.35), las competencias representan el resultado del proceso educativo, al constituir "capacidades individuales que permiten la realización de tareas u obtener ciertos logros en forma eficiente y eficaz. Entre sus componentes están los conocimientos, habilidades, destrezas, actitudes, rasgos personales", permitiendo al individuo interactuar en su accionar social. Es decir, que éstas son el resultado de experiencias integradoras de aprendizaje donde las destrezas, las habilidades y el conocimiento interactúan para generar aprendizaje que tiene valor de cambio en relación con la tarea para la cual fueron preparados.

Desde esa perspectiva, urge en la actual sociedad del conocimiento, la valoración e implementación de un nuevo currículo por competencias, que vincule a los diferentes niveles que integran el sistema educativo y sus respectivos métodos de evaluación, con base en sus exigencias y requerimientos establecidos por el ministerio de educación y los lineamientos internacionales de las organizaciones vinculadas al desarrollo de la praxis educativa. 
Evaluación de competencias. Retos y perspectivas desde el nivel básico al universitario

Díaz (2018), considera que el currículo por competencias implica asumir en el desarrollo curricular

cambios relevantes en las rutinas o demás formas de trabajo que añaden valor a los procesos pedagógicos y de organización que tienen lugar en el centro educativo; traducido en mejoras de los resultados de los aprendizajes de los estudiantes, en las formas cómo mejorar la realización de una tarea o actividad, o en ambas situaciones (par. 5).

En la misma línea de pensamiento, este proceso se canaliza por medio de la evaluación por competencias generadas en la praxis; que según Díaz y Hernández (2010, p. 8), involucra "un conjunto de actividades sistemáticas y necesarias dentro del proceso educativo que al ser administradas con estrategias pedagógicas y en ambientes adecuados, posibilitan recopilar, procesar y analizar un grupo de informaciones cumpliendo con requisitos metódicos, técnicos y científicos".

Es pertinente señalar, que a pesar de los esfuerzos realizados por entes internacionales no gubernamentales tales como Organización de las Naciones Unidas para la Educación, la Ciencia y la Cultura (UNESCO), Comisión Económica para América Latina (CEPAL), Organización de Naciones Unidas (ONU), Fondo Internacional de Emergencia de las Naciones Unidas para la Infancia (UNICEF), entre otros, articulado con el Ministerio de Educación Nacional (MEN) se evidencia el arraigo a modelos tradicionales centrados en la medición de contenidos cognitivos, en muchos de los casos descontextualizados, obviando las competencias actitudinales y proce-
Villero, $\mathrm{M}$ y cols.

dimentales desarrolladas por los estudiantes.

Ante está panorámica, se requiere de un cambio paradigmático que adopte una perspectiva constructivista, centrado en la obtención de resultados de aprendizajes contextualizados, apoyado en un perfil profesional y currículo por competencias en los diferentes niveles y su debido proceso de evaluación, acorde a las necesidades y objetivos institucionales.

Con base en la situación problema antes planteada, surge el presente estudio exploratorio documental, que se orientó en analizar la evaluación de competencias, retos y perspectivas desde los diferentes niveles educativos.

\section{DESARROLLO}

\section{Abordaje teórico para la comprensión del estudio}

En este aparte reflexivo se analizan teóricamente las categorías apriorísticas del estudio, para luego generar un proceso de triangulación que permita develar el accionar, desde la concepción de diferentes autores en relación con el constructo evaluación por competencias desde el nivel básico al universitario, sus retos y perspectivas en la nueva realidad social.

\section{Educación y competencias}

La educación y las competencias interactúan como un binomio en la actual sociedad del conocimiento, signada por vertiginosos cambios producto de los efectos de la globalización, pandemia, revolución 4.0. Esto significa una realidad compleja, donde los agentes involucrados en el hacer educativo cuentan con las herramientas y mecanismos disponibles en la era digital, para garantizar su conectividad. Se enfrenta, según Sánchez (2018) a un nuevo reto: 
Evaluación de competencias. Retos y perspectivas desde el nivel básico al universitario

lograr una educación a escala global que abarque las necesidades de formación del capital humano con la finalidad de generar una praxis educativa integral, desarrollando desde y fuera del aula, habilidades, competencias y conocimientos de utilidad no solo en un entorno local, sino también mundial (p.79).

Para tal efecto, el citado autor afirma, que la utilización de estas herramientas pedagógicas constituye un dispositivo transformador, ante la concepción negativa actual de valores sociales como: cooperación, solidaridad y consenso. No obstante, existen aspectos positivos del aprendizaje cooperativo y las estrategias dialógicas-comunicativas, vinculados a la integración e inclusión social, aunado al desarrollo de competencias fundamentales para superar barreras y problemas de convivencia en la actualidad. Ante esta exigencia, los sistemas educativos son llamados en la actualidad a realizar procesos de reingeniería organizacional abarcando aspectos curriculares, pedagógicos, tecnológicos, investigativos e innovando procesos de evaluación y control de sus acciones, ya que estos procesos deben ser evaluados con bases en las competencias desarrolladas cónsonas con la realidad y necesidades del recurso humano del país.

Al respecto, la UNESCO (2016), plantea en el objetivo 4 del desarrollo sostenible que la educación es la clave para poder alcanzar otros objetivos. Esta es fundamental para fomentar el desarrollo de competencias, tolerancia entre las personas, contribuyendo además a crear sociedades pacíficas orientadas a la búsqueda de estrategias novedosas y procesos cónsonos con la realidad social del momento.

Desde la perspectiva de los investigadores, los organismos gubernamentales respon-
Villero, M y cols.

sables de la educación deben articularse con las universidades como entes rectores de la praxis educativa y sus acciones de socialización en la formación de talento humano por competencias, requiriendo de un proceso de reingeniería desde los aspectos didácticos, presupuestarios, gerenciales, formativos, políticos, sociales, económicos, científicos y tecnológicos dando respuestas a las demandas sociales y su accionar en las comunidades emergentes de la era digital y del conocimiento.

En este caso se creyó pertinente, analizar la postura cognitiva y pragmática de La Secretaría de Educación Pública (como se citó en Becerril et al., 2015) al definir competencia como:

el desempeño que resulta de la movilización de conocimientos, habilidades, actitudes y valores, de un individuo, así como de sus capacidades y experiencias llevadas a cabo en un contexto específico, para resolver un problema o situación que se le presente en los distintos ámbitos de su vivir. (p.883).

En la misma línea del pensamiento, las competencias en el proceso educativo son clave para su estudio, ya que son el medio fundamental en la formación integral de los ciudadanos para el desarrollo de habilidades genéricas y técnicas, es necesario hacer una exhaustiva revisión y reflexión teórica sobre las competencias, al respecto Sandoval et al. (2008) plantean un sistema de enfoques teóricos sobre la evolución del concepto de competencia:

1. Enfoque funcionalista: donde se define a partir de un análisis de las funciones clave que cumple la persona dentro de su labor, con énfasis en los resultados o productos de la tarea, más que en cómo se logra hacerlos. "La principal 
Evaluación de competencias. Retos y perspectivas desde el nivel básico al universitario

crítica realizada, es que sólo verifica qué se ha logrado con una competencia, pero no cómo se logró, por lo que se dificulta la aplicación de la descripción de la competencia en los procesos formativos profesionales" (Prieto, 2007 como se citó en Sandoval et al., 2008, pp. 3-4).

2. Enfoque conductista: Está direccionado a la identificación de las habilidades personales (proponerse metas, tomar decisiones, detectar problemas y arriesgarse) que conllevan a desempeños de sus funciones dentro de la organización a la que pertenece. Desde este enfoque, "se considera a las competencias como comportamientos clave de las personas para la competitividad de las organizaciones" a juicio de Mertens (como se citó en Sandoval et al., 2008, p. 4).

3. Enfoque Constructivista: Desde esta concepción la competencia se vincula con los procesos organizacionales, concebida como "las habilidades, los conocimientos y las destrezas necesarias para resolver dificultades en los procesos laborales profesionales, desde el marco organizacional", según lo planteado por Tobón (como se citó en Sandoval et al., 2008, p. 6).

Atendiendo a esas consideraciones, para el estudio de la evaluación de competencias en los diferentes niveles del sistema educativo, es necesario tomar como referencia el enfoque constructivista como método colaborativo e innovador y conocer la taxonomía de las competencias partiendo de los enfoques de expertos como Leboyer (2002) y Becerril et al. (2015) quienes consideran que las más utilizadas en el ámbito organizacional son las genéricas y técnicas, las cuales se explican a continuación:
Villero, M y cols.

1. Competencias Genéricas: Para Leboyer (2002) son conductas identificadas y reforzadas en el ámbito empresarial para alcanzar éxito, enmarcado en un contexto determinado por la cultura y el estilo de trabajo de sus colaboradores. Estas deben poseerlas cada trabajador o miembro de la organización, indistintamente de la función o cargo que desempeñen.

Enuncia el citado autor, un listado entre las más importantes:

Adaptabilidad, ambición, amplitud de espíritu, autonomía, autoridad, capacidad de concentración, capacidad de mando, capacidad de síntesis, confianza en sí mismo, control en sí mismo, coordinación, creatividad, disciplina, energía, expresión escrita, expresión oral, identificación y análisis de problemas, persuasión, razonamiento y resolución de problemas, sensibilización social, sentido de la negociación, sociabilidad, tolerancia. (p.57)

2. Competencias Técnicas: A juicio de Becerril et al. (2015, p.884) "engloban un conjunto de conocimientos específicos necesarios (know how) para el desarrollo de las actividades propias del área". Éstas se identifican y se definen dependiendo en cada área funcional.

En el marco de las exigencia de la realidad actual, se requiere desarrollar estas competencias vinculadas a las habilidades virtuales, que de acuerdo con Vilchis (2014) se encuentran: (a) Demostrar conocimiento relacionado con la informática, (b) Apropiarse de teorías y prácticas de la ingeniería informática para ser aplicadas en el área de trabajo, (c) Seleccionar tecnología de punta, evaluando 
Evaluación de competencias. Retos y perspectivas desde el nivel básico al universitario

su aplicabilidad para satisfacer a los clientes o usuarios, (d) Evaluar sistemas hardware/ software en función de un criterio de calidad determinado por la organización, (e) Conocer los requisitos de un sistema informático para poder alcanzar los objetivos tanto organizacionales como personales; y, (f) Generar y desarrollar proyectos de tecnología de información y comunicación, utilizando las metodologías acordes a los objetivos institucionales.

Desde la perspectiva de los investigadores, es fundamental en el actual momento, la capacitación digital de la comunidad educativa para darle respuesta a los escenarios complejos y adaptarse a los estándares de la sociedad del conocimiento y el desarrollo sostenible del nuevo orden social.

Además, se requiere darles seguimiento, vinculación y continuidad curricular a los procesos educativos de los diferentes niveles, aplicando herramientas y técnicas que conjuguen motivación, innovación, creatividad, investigación en un modelo educativo constructivista donde el docente combine las competencias genéricas y técnicas para el logro de los objetivos institucionales y las necesidades del talento humano requeridas por el país.

\section{Enfoque curricular por competencias}

Es importante tener presente dentro del sistema educativo, la articulación de los niveles educativos, tomando en cuenta los perfiles de egreso y los diseños curriculares basados en competencias como aspectos primordiales en brindar una educación pertinente y de calidad. Al respecto, González (2009) asevera que este tipo de currículo:

es un documento elaborado a partir de la descripción del perfil profesional, es decir, de los desempeños esperados de
Villero, $\mathrm{M}$ y cols.

una persona en un área ocupacional, para resolver los problemas propios del ejercicio de su rol profesional. Procura de este modo asegurar la pertinencia, en términos de empleo y empleabilidad, de la oferta formativa diseñada (p.54).

En la misma línea de pensamiento, Díaz et al (2008) refieren que "el currículo es irreducible al documento porque todo plan es transformado por efecto de la acción que él mismo engendra". Por lo tanto, todo accionar humano lleva a ser transformado por los objetivos que lo inspiraron, dado que las personas como vivientes mutables no son programables; por consiguiente, "la variabilidad humana es absolutamente preferible a la uniformidad de la conducta individual y colectiva".

En consecuencia, señalan los citados autores que,

ese efecto retroactivo actúa siempre y es la justificación de la evaluación curricular, que debe velar porque permanezcan los valores universales inmanentes, al tiempo que cambia $y$ modifica el currículo para ponerlo a tono con los avances sociales, científicos y tecnológicos. En pocas palabras, el currículo más que una prescripción es una hipótesis flexible, susceptible de modificarse por la participación de los docentes, estudiantes, otros agentes educacionales y la retroacción que causa la misma acción educativa (p. 51).

En suma, tomando en cuenta las Interpretaciones realizadas al término currículo, se evidencia que se han considerado diferentes criterios, con base a la literatura especializada antes mencionada, donde se concibe el currículo de diversas maneras. Así, por 
Evaluación de competencias. Retos y perspectivas desde el nivel básico al universitario

ejemplo, si nos atenemos a sus momentos de concepción y aplicación el currículo es plan, proceso y resultado. O más bien, se puede concluir que el currículo es algo que se desenvuelve como documento (teoría, formal), como acción educacional (proceso, praxis, práctico aplicado) y como resultado de aprendizaje educativo (producto, encarnado en la personalidad de estudiantes y docentes).

\section{Evaluación en el sistema educativo}

El contexto impuesto por la globalización, la pandemia del Covid-19 y la revolución 4.0 ha generado nuevas demandas a todo el sistema educativo y con ello, la necesidad de transformar y repensar las prácticas pedagógicas y evaluativas aplicadas en los ambientes educativos. En este sentido se requiere una articulación de los saberes, habilidades y destrezas desde una óptica holística e integradora con las competencias que las personas deben poseer para enfrentar el nuevo orden.

La evaluación educativa urge de cambios del proceso tradicional presencial a lo virtual a distancia con modificaciones presenciales que según, Díaz y Hernández (2010, p. 95) involucra "un conjunto de actividades sistemáticas y necesarias dentro del proceso educativo que al ser administradas con estrategias pedagógicas y en ambientes adecuados, posibilitan recopilar, procesar y analizar un grupo de informaciones cumpliendo con requisitos metódicos, técnicos y científicos".

En correspondencia a lo expuesto la Universidad Internacional de la Rioja (UNIR, 2020) concibe como institución de educación superior a la evaluación como

un proceso continuo y personalizado dentro del sistema de enseñanza-aprendizaje cuyo objetivo es conocer la evo-
Villero, M y cols.

lución de cada estudiante con el fin de adoptar medidas de refuerzo o de compensación para garantizar que se alcanzan los objetivos educativos definidos para su nivel. Es, por tanto, una herramienta de gran utilidad para tomar decisiones pedagógicas para mejorar el desempeño de un estudiante (parr. 2).

Desde esa perspectiva, la evaluación no solo es útil para los profesores, sino también para los estudiantes. A nivel profesoral se les posibilita comunicar a los estudiantes los objetivos y expectativas de aprendizaje; comprobando así, la eficacia de los métodos de enseñanza aplicados. En cuanto a los estudiantes, este proceso sirve como incentivo a su esfuerzo por medio de valoraciones, implicando la retroalimentación de los conocimientos previos (Consolidación de saberes).

La importancia de realizar una evaluación en educación, de acuerdo con la UNIR (2020, parr. 4) "va más allá del seguimiento escolar de los propios estudiantes, se trata de un instrumento de seguimiento y valoración de los resultados obtenidos por los escolares para poder determinar si los procedimientos y metodologías educativas elegidas son los adecuados". Es decir, la evaluación educativa no implica solo aplicar pruebas, sino que es un proceso integrador donde intervienen todos los agentes involucrados en el hecho educativo (docentes, planteles, currículos, planes, programas, proyectos y la gestión administrativa en general).

Para Stake (como se citó en Núñez 2017), "los evaluadores conjuguen dos formas distintas de pensar y dos paradigmas de investigación que anteriormente no era buena opción, pero hoy día, es excelente combinación". Se les suele denominar cualitativo (comprensivo e interpretativo) y cuan- 
Evaluación de competencias. Retos y perspectivas desde el nivel básico al universitario

titativo (basado en estándares o criterios).

Al respecto argumentan los investigadores, que el rol de evaluador y facilitador de aprendizajes del docente son dos componentes fundamentales en su praxis educativa, que le permiten llegar a conocer a cabalidad a sus alumnos, ¿cómo es su proceso de aprendizaje? en consecuencia, tomar decisiones acertadas para acompañarlos en el accionar de las competencias requeridas en su transitar social. Sin embargo, el sistema habitual de evaluación debe contemplar además del plan de estudio, los objetivos, contenidos, estrategias didácticas y de evaluación; aunado al grado de adquisición de las competencias exigidas para cada nivel educativo.

\section{Evaluación por competencias a nivel básico}

Acorde con las propuestas y exigencias actuales en el contexto educativo, el proceso evaluativo en las aulas de clase se concibe de acuerdo con Prieto y Contreras (2008, p. 246) "como un proceso complejo orientado a recoger evidencias respecto del aprendizaje de los estudiantes de manera sistemática, para emitir juicios en pos de un mejoramiento tanto de la calidad del aprendizaje como de la enseñanza". Asimismo, al realizar la evaluación se distinguen varios sistemas o técnicas utilizados de manera complementaria en el nivel básico para valorar las competencias. Al respecto, UNIR (2020) y Núñez (2017) se tienen: la observación, pruebas sumativas y formativas (escritas de respuestas estructuradas, semiestructuradas y no estructuradas) revisión de tareas, ensayos, mapas conceptuales, entrevistas, evaluación de actitudes y personalidad, actividades individuales y grupales, entre otras.

Todo este proceso se encuentra enmarcado en los cuatros pilares de la educa-
Villero, M y cols.

ción "aprender a conocer, aprender a hacer, aprender a vivir juntos y a convivir con los demás y aprender a ser" propuesto por Delors (1996) en el documento "la educación encierra un tesoro" presentado en el informe de la UNESCO de la Comisión Internacional sobre la Educación para el siglo XXI.

Desde esa perspectiva, la relación del binomio praxis docente-práctica evaluativa, busca precisar nuevas demandas en la formación de competencias con el fin de "superar patrones limitantes, legitimar el logro del aprendizaje complejo e involucrar diversidad de saberes", a criterios del autor antes citado:

1. Aprender a saber: se refiere a la adquisición de capacidades para conocer y comprender el mundo; se relaciona con la competencia de "aprender a aprender".

2. Aprender a hacer: se centra en ejercer influencia en el entorno inmediato, hacer enfrentando las vicisitudes y problemas, aplicando la teoría aprendida.

3. Aprender a convivir: implica poseer las habilidades sociales para vivir con los demás en un ambiente de sana convivencia. Esto exige entender y respetar al prójimo, manifestando los valores sociales.

4. Aprender a ser: consiste en desarrollar potencialidades individuales, desarrollando la autonomía y en efecto, su capacidad de juicio y responsabilidad. Este pilar de la educación, cumple con la función de integrar todos los saberes antes mencionados.

Visto así, se requiere saber de acuerdo con el informe emanado de la Comisión Interna- 
Evaluación de competencias. Retos y perspectivas desde el nivel básico al universitario

cional sobre la Educación para el siglo XXI,

cómo evaluar los conocimientos asociados al aprendizaje complejo; intervenir con procedimientos claros (saber hacer) realizar un recorrido metodológico; poseer el conocimiento condicional, con una actitud asociada al entorno humanista y ético de la evaluación situada en la interacción con otros (saber ser) y una actitud sostenida de la evaluación para el aprendizaje (saber estar) de manera trascendente y eficaz, como profesor, profesional competente y comprometido (parr. 11).

\section{Evaluación por competencias a nivel uni- versitario}

El contexto universitario, ha pasado por diferentes enfoques evaluativos en la praxis educativa, donde la globalización, la revolución 4.0 y más reciente la pandemia han generado nuevas demandas a todo el sistema educativo y con ello, la necesidad de innovar y reformular las prácticas pedagógicas y evaluativas. Desde esa óptica, los investigadores y teóricos expresan que las competencias surgen como una respuesta a la necesidad de articular los saberes desde su carácter holístico e integrado con las capacidades que los sujetos deben poseer para enfrentar el mundo laboral.

Al respecto, Ríos y Herrera (2017, p. 1076) plantean que

las competencias emergieron como una respuesta capaz de enfrentar las relaciones entre lo académico, los aprendizajes y los desafíos laborales de los sujetos. Sin embargo, la duda es cómo transformar estos nuevos contextos en rediseños o formulaciones innovadoras en las prácticas evaluativas que trabajen con las
Villero, $\mathrm{M}$ y cols.

competencias como un objeto evaluativo. Para Jaimes y Callejas (Como se citó en Ríos y Herrera 2017, p. 1078), la evaluación debe contemplar los pilares del saber (el saber, el saber hacer y el saber ser), punto en el que se interceptan especialmente los diferentes aprendizajes desde una mirada transversal, ya que estos se posicionan y construyen desde la cotidianeidad educativa.

En la misma línea del pensamiento, Tobón (2006, p. 78) plantea que "la evaluación de las competencias debe integrar lo cualitativo con lo cuantitativo, pues con palabras no se puede medir, y con números no se puede comprender ni explicar", en ese sentido ambos aspectos contribuyen a mejorar la praxis educativa. Desde esta perspectiva a nivel universitario, la evaluación por competencias posee un valor multidimensional, ya que, la evaluación por competencias se centra en la efectividad de los planes de estudios, el desarrollo de los objetivos institucionales de la praxis y el logro de los mismos vinculado al perfil de egreso.

Asimismo, García et al. (como se citó en Ríos y Herrera, 2017, p.1079) establecen los mismos principios que

la evaluación por competencias requiere un modelo de evaluación propio, en el sentido que se preocupe por esquematizar en forma clara y sintética, patrones y componentes evaluativos, considerando su representación arquetípica, procesos de retroalimentación, funciones de los agentes involucrados y secuencias concretas que evidencien los desarrollos de los aprendizajes logrados.

En atención a esta postura epistémica, la evaluación por competencias para los investigadores resume un proceso de aprendizaje 
integral con énfasis en el alcance holístico de los estudiantes y no en el resultado. Es decir, entre los aspectos más significativo del medio educativo se destaca la evaluación en la educación universitaria, debido a su relación e inmersión en la construcción de los agentes que liderarán y transformarán las sociedades.

Asevera Terán (2019) que la evaluación se elabora y se pone en práctica de acuerdo con la intención del sujeto que se busque formar, tomando como base los diversos criterios que se establecen como puntos de referencia, para apoyar y facilitar la toma de decisiones al final de proceso; además, durante la formación el docente debe apropiarse de procedimientos e instrumentos que soporten el seguimiento de la enseñanza y aprendizaje.

Al respecto, el autor referido, establece la importancia de los aspectos formativos del proceso evaluativo, enfocado más en la comprensión que la reproducción, además posibilita la forma de construir y reconstruir el conocimiento con los estudiantes, es decir, generando espacios de participación y discusión.

En relación con lo mencionado, el proceso de evaluación en síntesis abarca dos elementos fundamentales como lo son; los criterios de evaluación y la recolección de información, evidenciándose en las pruebas escritas, trabajos, disertaciones, entre otros instrumentos aplicado por el docente con la finalidad de obtener evidencias a través de la observación de las interacciones dadas en las aulas.

En suma. para los investigadores, las competencias a nivel universitario son saberes combinados entre los pilares del saber de la educación donde se vincula el ser, el saber hacer y el saber estar, para obtener competencias conceptuales, procedimentales y actitudinales para interactuar en los contextos educativos y laborales, controlando las acciones y efectos sobre la praxis educativa y la evaluación del mismo con el fin de garantizar el éxito académico e institucional. (competencias conceptuales, psicopedagógicas, metodológicas y sociales).

\section{Retos y perspectivas en evaluación por competencias a nivel básico y universitario.}

Con base en las reflexiones críticas sobre el análisis documental de la evaluación por competencias en los niveles básico al universitario como variable clave en la educación, se evidenció información valiosa para optimizar la situación problemática y posibles lineamientos, asumiendo los referentes teóricos para la acción transformadora y las categorías emergentes construidas por los investigadores en el proceso de triangulación, con la finalidad de interpretar la realidad develada para su transformación y generar acciones ante el nuevo orden social.

Estos lineamientos se proponen basándose en los postulados teóricos de los autores que han sido analizados, apoyándose además en las líneas estratégicas propuesta por organismos internacionales vinculado a la praxis educativa entre los retos y perspectivas más importantes se tienen:

1. La praxis educativa del nuevo orden social debe estar planificada bajo los estándares de la ONU, UNESCO, CEPAL, UNICEF, OMS entre otras organizaciones internacionales con principios y lineamientos éticos, inclusivos, de calidad, de bioseguridad, nuevas tecnologías de información y comunicación respetando los objetivos del desarrollo sostenible para 2030 articu- 
lando ciencia, tecnología, innovación e investigación basado en competencias contemplando los pilares del saber.

2. Solicitar a los gobiernos prioridad al ámbito de la educación en el diseño de las políticas y las prácticas educativas, investigativas e innovación articulando el currículo por competencias y su proceso de evaluación en los diferentes niveles educativo.

3. Alfabetización digital y curricular por competencias al personal docente como estrategia fundamental en los escenarios educativos mundiales.

4. Articulación curricular por competencias de los diferentes niveles del sistema educativo cónsona con los requerimientos y necesidades del país haciendo énfasis en la población más vulnerable y proporcionando una educación gratuita e inclusiva.

5. Conformar equipos multidisciplinarios con entes del gobierno, universidades, sector privado $\mathrm{y}$ sociedad en busca de alternativas financieras y de redimensión curricular por competencias que garanticen una educación de calidad e inclusiva.

6. Generar alianzas estratégicas con las empresas privadas creando convenios de inversión, dotación y atención a las instituciones educativas fomentando nuevas e innovadoras herramientas pedagógicas basadas en competencias tecnológicas.
7. Adecuar los perfiles de egreso a las necesidades y lineamientos curriculares por competencias cónsono con el nuevo orden social.

8. Centrar la praxis educativa en la evaluación por competencias como un modelo que abarque procesos y procedimientos, vinculados a los pilares del saber, cuyo norte sea alcanzar resultados de aprendizajes.

9. Articular lo académico y laboral como una arista más para el desarrollo de las competencias de manera integral, acorde a las necesidades y demandas del país.

10. Propiciar la innovación tecnológica como una visión integradora que busque la formación holística de ciudadanos críticos y preparados ante el nuevo orden social. En síntesis, el enfoque curricular basado en competencias permite buscar nuevas respuestas y horizontes al desafío profesional en un mundo signado por la tecnología de la información $\mathrm{y}$ comunicación [TIC], en un contexto educativo que requiere la vinculación del binomio ciencia y tecnología con la finalidad de producir cambios sociales y la formación holística de las personas.

La Investigación desarrollada se fundamentó en un paradigma cualitativo que según Hernández y Mendoza (2018, p. 9) plantea que "trata de identificar, básicamente, la naturaleza profunda de realidades, su estructura dinámica, aquella que da razón plena de su comportamiento y manifestaciones, producto de técnicas 
para recabar información, como la revisión de documentos y su interpretación". La misma, se apoyó en un nivel exploratorio que de acuerdo con Muñoz (2018) identifica conceptos promisorios para indagar, preparando el terreno para estudios más amplios, elaborados y profundos.

En la misma dirección metodológica, se asumió la modalidad de revisión documental que según criterios de Ortiz (2015, p. 30) "se centra en el estudio de problemas con el propósito de ampliar y profundizar el conocimiento de su naturaleza, con apoyo, principalmente, en trabajos previos, información y datos divulgados por medios impresos o electrónicos". Además, se empleó la técnica análisis de contenido con base en la estructura semántica, que a juicio de Álvarez (2016, p.163) es "uno de los procedimientos que más se acerca a los postulados cualitativos desde sus propósitos; busca analizar mensajes, rasgos de personalidad, preocupaciones y otros aspectos subjetivos."

Siguiendo con esa secuencia operativa, se utilizó un diseño bibliográfico que para Palella y Martins (2017, p.87), se fundamenta “en la revisión sistemática, rigurosa y profunda del material documental de cualquier clase. Se procura el análisis de los fenómenos o el establecimiento de la relación entre dos o más categoría". Cuando los investigadores, optan por este tipo de estudio, el utilizan documentos, los recolectan, seleccionan, analizan y presentan resultados coherentes", a fin de develar las discusiones generadas en el escenario sobre la evaluación de competencias. Retos y perspectivas desde el nivel básico al universitario. A partir de allí, se cristalizó la reflexión teórica objeto de estudio con el fin de recopilar y actualizar información sobre la temática generando discusión y los alcances en acciones emergentes en la nueva realidad social.

\section{CONCLUSIONES}

Al analizar la evaluación de competencias, retos y perspectivas desde el nivel básico al universitario se develó:

El modelo basado en competencias facilita la formación profesional de los estudiantes, debiendo estar centrado en aspectos paradigmáticos de la política y el diseño curricular educativo.

El enfoque curricular basado en competencias se centra en el perfil de egreso de los estudiantes, lo que permite vincularlos con las necesidades de la región, del país y de la institución formadora.

Las instituciones educativas, asumen un enfoque de evaluación constructivista contextual basado en competencias, lo cual implica centrar la praxis educativa en un modelo orientado en la evaluación de los procesos y procedimientos.

Existen docentes que mantienen el uso de prácticas evaluativas tradicionales centradas en medir solo la dimensión cognitiva, obviando la concepción integradora propuesta por organismos internacionales y asumida por el Ministerio de Educación Nacional.

Desde la perspectiva de los investigadores, se requiere reformular y reforzar los procesos educativos vinculados al desarrollo de habilidades y competencias de los estudiantes, con la finalidad de prepararse para el cambio con la utilización de recursos enfocados en la toma de decisión.

En estos tiempos de cambios, es fundamental enfrentar los avatares de la globalización, 
pandemia, revolución industrial 4.0 y la construcción del conocimiento, basado en gran brecha económica, promoviendo la una educación holística por competencias.

\section{REFERENCIAS BIBLIOGRÁFICAS}

Álvarez, J. (2016). Cómo hacer investigación cualitativa. Fundamentos y metodologías. Paidós Educador. México.

Becerril, C., Sosa, G., Delgadillo, M. y Torres, S. (2015). Competencias Básicas de un Docente Virtual. Revista de Sistemas y Gestión Educativa. 2(4), pp. 882-887. https://www.ecorfan.org/bolivia/researchjournals/Sistemas_y_Gestion_Educativa/vol2num4/18.pdf.

Delors, J. y otros (1996). La educación encierra un tesoro. Santillana, ediciones UNESCO. https://unesdoc.unesco.org/ark:/48223/pf0000109590_spa.

Díaz, F, Lule, M., Pacheco, D., Rojas, S., y Saád, E. (2008). Metodología de diseño curricular para educación superior. Trillas: México.

Díaz, H. (2018). Innovación curricular. http://educared.fundaciontelefonica.com.pe/desafioseducacion/2018/10/09/innovacion-curricular/.

González, M. (2009). Currículo basado en competencias: una experiencia en educación universitaria, D - Universidad de La Sabana. ProQuest Ebook Central, http://ebookcentral.proquest.com/lib/biblioupcsp/detail.action?docID=3185119.

Jaimes, G y Callejas, M. (2009). La autonomía, los procesos de pensamiento y las TIC: competencias del siglo XXI. Temática contemporánea de aplicación en escuelas, colegios y universidades. Colombia.

Hernández, R y Mendoza, C. (2018). Metodología de la investigación. Las rutas cuantitativa, cualitativa y mixta. Editorial McGrawHill Education. México. DOI: $10.36192 / 978-3-948768003$

Leboyer, C. (2002). Gestión de las competencias. Cómo analizarlas, cómo evaluarlas, cómo desarrollarlas. Editorial Gestión 2000.

Mertens, L. (2002). ISO 9000:2000 y Competencia laboral: El aseguramiento del aprendizaje continuo e incluyente en la organización. Montevideo: CINTERFOR/OIT. http://www.leonardmertens.com/showarticle.php?id=22\&backurl=section \% 253D1\%2526start\%253D30. 
Muñoz, C. (2018). Metodología de la investigación. Ciencias sociales. Oxford. México.

Nuñez, K. (2017). Evaluación de los aprendizajes sobre ciudadanía: meta evaluación de los instrumentos utilizados en el segundo ciclo básico chileno. https://scielo.conicyt.cl/scielo.php?pid=S0718-07052017000200014\&script=sci_arttext\&tlng=n.

Organización de las Naciones Unidas para la Educación, la ciencia y la Cultura - UNESCO. (2016). Desglosar el Objetivo de Desarrollo Sostenible. Educación 2030. https://es.unesco.org/sdgs.

Ortiz, A. (2015). Enfoques y métodos de investigación en las ciencias sociales y humanas. Ediciones de la U. Bogotá, Colombia.

Palella, S. y Martins, F. (2017). Metodología de la investigación cuantitativa. Fondo editorial de la Universidad Pedagógica Experimental Libertador. Venezuela.

Prieto A. (2007). "Identificación y análisis de competencias: enfoque conductista, constructivista y funcionalista Breve descripción de los 3 enfoques y su aplicación práctica” en Trabajadores competentes - Ed. ESIC.

Prieto, M. y Contreras, G. (2008). Las concepciones que orientan las practicas evaluativas de los profesores: un problema a develar. Revista Estudios Pedagógicos. XXXIV (2), 245-262. https://scielo.conicyt.cl/pdf/estped/v34n2/art15.pdf.

Ríos, D y Herrera, D. (2017). Los desafíos de la evaluación por competencias en el ámbito educativo. Educ. Pesqui., São Paulo, v. 43, n. 4, p. 1073-1086. Universidad de Santiago de Chile, Santiago, Chile. http://dx.doi.org/10.1590/S1678-4634201706164230.

Rojas, M. (2016). Gestión educativa en la sociedad del conocimiento. Editorial Magisterio. Bogotá, Colombia.

Sandoval, F., Miguel, V., y Montaño, N. (2008). "Evolución del concepto de competencia laboral", http://www.ucv.ve/-leadmin/user_upload/vrac/documentos/Curricular_Documentos/ Evento/Ponencias_6/sandoval_Franklin_y_otros.pdf.

Terán, V. (2020). Concepciones y prácticas evaluativas de docentes en programas de pregrado de la Universidad Católica Luis Amigó en Montería Colombia. Tesis doctoral. Universidad Metropolitana de Educación, Ciencia y tecnología. UMECIT. https://repositorio.umecit.edu.pa/bitstream/handle/001/2933/TVictor\%20Teran.pdf?sequence $=1 \&$ isAllowed $=\mathrm{y}$. 
Tobón, S. (2006). Formación basada en competencias: pensamiento complejo, diseño curricular y didáctica. ECOE, Colombia.

Universidad Internacional de La Rioja [UNIR] (2020). Evaluación educativa: en qué consiste, importancia y sistemas habituales empleados para evaluar sistema. https://www.unir.net/educacion/revista/noticias/evaluacion-educativa/549205088963/.

Vilchis, L. (2014). Pensar en las artes y el diseño: Estrategias de evaluación del proyecto. https://luzdelcarmenvilchis.blogspot.com /2014/05/estrategias-de-evaluacion-del-proyecto.html. 